\title{
An unusual p53 mutation detected in Burkitt's lymphoma: 30 bp duplication
}

\author{
JANA SMARDOVA ${ }^{1}$, DIANA GROCHOVA ${ }^{1}$, PAVEL FABIAN $^{2}$, MOJMIR MOULIS $^{1}$, JAN SMARDA $^{3}$, \\ IVA FALKOVA ${ }^{1}$, BARBORA RAVCUKOVA ${ }^{1}$, JANA VANKOVA ${ }^{1}$ and INGRID VASOVA ${ }^{4}$ \\ ${ }^{1}$ Department of Pathology, University Hospital Brno, Jihlavska 20, 62500 Brno; ${ }^{2}$ Masaryk Memorial Cancer Institute, \\ Zluty kopec 7, 65653 Brno; ${ }^{3}$ Department of Experimental Biology, Faculty of Science, Masaryk University, \\ Kotlarska 2, 61137 Brno; ${ }^{4}$ Department of Internal Hematooncology, University Hospital Brno, Czech Republic
}

Received February 25, 2008; Accepted May 22, 2008

DOI: 10.3892/or_00000073

\begin{abstract}
Burkitt's lymphomas (BL) are aggressive rapidly growing tumors typified by a high c-myc expression resulting from $\mathrm{t}(8 ; 14)(\mathrm{q} 24 ; \mathrm{q} 32), \mathrm{t}(2 ; 8)(\mathrm{p} 12 ; \mathrm{q} 24)$ or $\mathrm{t}(8 ; 22)(\mathrm{q} 24 ; \mathrm{q} 11)$ translocations. Alterations of the p53 tumor suppressor are also relatively frequent in BL. Several approaches have been adopted for detection of the p53 aberrations such as immunohistochemical analyses, immunoblotting, DNA sequencing, fluorescence in situ hybridization (FISH), and functional assays. We used these methods to characterize the p53 mutation in tumor cells of a 53-year-old male suffering from Burkitt's lymphoma. By immunohistochemical analyses, we detected high levels of the p53 protein in the tumor tissue. Immunoblotting showed a higher molecular weight of the p53 protein overexpressed in the tumor tissues than that of the standard p53 protein. Similarly, the molecular weight of the PCR product prepared by amplification of the tumor p53 cDNA was higher than that of the standard p53 cDNA. Functional analyses of separated alleles in yeast evidently revealed that the tumor $\mathrm{p} 53$ protein was transcriptionally nonfunctional. The yeast colonies expressing this p53 variant possessed a unique phenotype in that they were red with many white spots on their surface. Sequencing of the tumor cDNA revealed a duplication of the $30 \mathrm{bp}$ region of the p53 gene (g.12155_12184dup30) leading to a repeat of 10 amino acids (Pro-77 to Ala-86) in the p53 protein. Further analyses showed that the mutation was unstable in yeast cells. The FISH analyses did not confer loss of the p53-specific locus $17 \mathrm{p} 13$.
\end{abstract}

\section{Introduction}

Burkitt's lymphomas (BL) are aggressive rapidly growing tumors typified by the high expression of the c-myc proto-

Correspondence to: Dr Jana Smardova, Department of Pathology, University Hospital, Jihlavska 20, 62500 Brno, Czech Republic E-mail: janasmarda@seznam.cz

Key words: Burkitt's lymphoma, p53 tumor suppressor, functional analysis of separated alleles in yeast, duplication oncogene; a phenomenon resulting from the reciprocal chromosomal translocation $\mathrm{t}(8 ; 14)(\mathrm{q} 24 ; \mathrm{q} 32), \mathrm{t}(2 ; 8)(\mathrm{p} 12 ; \mathrm{q} 24)$ or $\mathrm{t}(8 ; 22)(\mathrm{q} 24 ; \mathrm{q} 11)$ activating $\mathrm{c}-m y c$ through its juxtaposition to one of the immunoglobulin loci. Overexpressed c-Myc then provides a constitutive proliferative signal and initiates apoptosis due to the p14ARF-dependent activation of p53. Studies suggest that inactivation of the p14ARF-MDM2-p53 apoptotic signaling pathway represents an essential secondary event in the development of BL. A significant proportion of BL cases carry point mutations in the p53 tumor suppressor gene or other defects in the p14ARF-MDM2-p53 pathway (1-3).

The p53 tumor suppressor is a sequence-specific transcriptional factor controlling the expression of multiple target genes, and participating in the regulation of many cellular processes, such as cell cycle, apoptosis, senescence and genome stability (4). Inactivation of p53 by various genetic alterations is a major event in human tumorigenesis. More than $50 \%$ of human tumors exhibit a mutation in the p53 gene. More than $80 \%$ of the p53 alterations are missense point mutations that lead to the synthesis of a stable full-length protein $(5,6)$. Several different approaches have been adopted for detection of the p53 aberrations. i) In normal cells, the p53 protein level is largely determined by the MDM2-mediated degradation. The MDM2 gene expression is controlled by p53, providing a negative feedback regulatory loop (7). Inactive p53 fails to induce MDM2 expression, and is stabilized in cell nuclei. The p53 protein level can be determined either on tissue sections by immunohistochemical analysis or on nitrocellulose membranes after protein gel electrophoresis and immunoblotting. ii) There are multiple types of molecular analyses for detection of changes in nucleotide sequences of genome DNA, mRNA and cDNA. Among these approaches, direct DNA sequencing is the most reliable method of detecting mutations in the p53 gene. However, the sensitivity of this method is rather low when tumor tissues are contaminated with normal cells. iii) Functional assays can specifically target some biological features of the p53 protein. The functional analysis of separated alleles in yeast (FASAY) is based on evaluation of the trans-activational capability of the tumor-derived p53 protein that is produced from amplified tumor p53 cDNA in yeast cells (8). Activity of the 
p53 protein is determined according to the color of the yeast colonies equipped with the p53-responsive RGC sequence upstream of the ADE2 reporter gene. Functional p53 causes the formation of white yeast colonies while mutant p53 is marked by red colonies (9). iv) According to the classic twohit hypothesis, the two alleles of the majority of tumor suppressors undergo inactivation. This feature has been repeatedly documented for p53 (10). The most common but not exclusive way of loss of p53 heterozygosity is loss of the p53-specific locus on chromosome 17 (17p13.1). Fluorescence in situ hybridization (FISH) using the $\mathrm{p} 53$-specific probe is a convenient method to detect this type of p53 aberration.

We employed the above-mentioned methods to characterize an unusual p53 mutation detected in tumor tissue of the BL patient in detail. In this case, the p53 protein was enlarged and functionally abrogated due to the $30 \mathrm{bp}$ duplication, g.12155_12184dup30 of the p53 gene leading to a repeat of 10 amino acids (Pro-77 to Ala-86) in the p53 protein. The duplicated p53-coding sequence represents the fragment limited by codons 77 and 87 .

\section{Materials and methods}

Case report. A 53-year-old male underwent explorative abdominal surgery in April 2006. The patient was suffering from lymphoma with extensive infiltration of the abdominal cavity, stomach, surrounding lymph nodes, bone marrow and central nervous system. The disease was diagnosed as classic Burkitt's lymphoma, clinical stage IVB, featuring the $\mathrm{t}(8 ; 14)$ translocation detected by FISH. The patient underwent an intensive combined immunochemotherapy and achieved complete remission, which has lasted up to the present date.

Histopathology. The adipose component of excised tissues was diffusely infiltrated by neoplastic populations of relatively uniform medium-sized lymphoid cells. Nuclei of the neoplastic cells were spherical or polygonal and contained dispersed chromatin and several (mostly 2-3) visible small nucleoli. The cytoplasm was basophilic. Mitotic activity was extremely high as well as the rate of apoptosis. A typical starry sky pattern was detected. Occasional reactive small round $\mathrm{T}$ lymphocytes were admixed. The tumor cells were immunohistochemically negative for CD3, CD5, CD23, CD30 and BCL2; positive for CD10, CD20, CD45, CD79a, BCL6 and MUM 1; and focally for CD138. The Ki67 proliferation index reached $95 \%$.

FASAY. FASAY was performed as described earlier $(9,11)$. Total RNA was purified using RNeasy mini kit (Qiagen Inc., Valencia, CA, USA). cDNA was synthesized by SuperScript II (Invitrogen, Corp., Carlsbad, CA, USA) using oligo(dT) $)_{12}$ as a primer. PCR was performed using primers P3 (5'-CCTTGC-CGT-CCC-AAG-CAA-TGG-ATG-AT-3'), P4 (5'-ACCCTT-TTT-GGA-CTT-CAG-GTG-GCT-GGA-GT-3'), and Pfu DNA polymerase (Stratagene, La Jolla, CA, USA). Yeast cells were co-transformed with the PCR product, linearized pSS16, and the salmon sperm DNA carrier (Invitrogen) by the lithium acetate procedure (8). Transformed yeast cells were plated on minimal medium lacking leucine and with a low amount of adenine $(5 \mu \mathrm{g} / \mathrm{ml})$, followed by incubation at $35^{\circ} \mathrm{C}$ for $2-3$ days, and then for 2-3 days at room temperature.
Recovery of plasmid from yeast and DNA sequencing. Yeasts harboring the p53 plasmid were lysed with lysing enzymes of Trichoderma harzianum (Sigma-Aldrich, Prague, Czech Republic) and the p53 expression plasmid was extracted. The central part of the p53 gene was amplified by PCR using P3 and $\mathrm{P} 4$ primers and Taq polymerase (Invitrogen). The PCR product was extracted from a gel by the MinElute PCR purification kit (Qiagen) and sequenced by BigDyeTerminator v3.1 cycle sequencing kit (Applied Biosystems, Foster City, CA, USA) using an ABI PRISM 310 genetic analyzer (Applied Biosystems).

Immunohistochemical analysis. Endogenous peroxidase activity was blocked with $3 \%$ hydrogen peroxide in methanol, for $10 \mathrm{~min}$. Antigen retrieval was performed in citrate buffer, pH 6.0 (Dako Denmark A/S, Glostrup, Denmark) at $121^{\circ} \mathrm{C}$ for 4 min. The p53-specific antibody DO-7 (Dako Denmark A/S) diluted 1:2000 was applied at $4^{\circ} \mathrm{C}$ overnight. Reactive sites were identified using biotinylated secondary antibody, peroxidase ABC (Vector Laboratories, Burlingame, CA, USA), DAB (Dako Denmark A/S), and were counterstained with Gill's haematoxylin. Optimal antibody concentration was determined as the highest dilution that demonstrates positive staining in normal squamous epithelium of the uterine cervix.

Immunoblotting. Tissue was lysed with buffer containing $150 \mathrm{mM} \mathrm{NaCl}, 50 \mathrm{mM} \mathrm{NaF}, 50 \mathrm{mM}$ Tris $(\mathrm{pH} 8.0), 5 \mathrm{mM}$ EDTA, 1\% NP40 and $1 \mathrm{mM}$ phenylmethylsulfonylfluoride protease inhibitor in ice for $30 \mathrm{~min}$, and the cell extract was centrifuged at $17000 \mathrm{~g}$ for $30 \mathrm{~min}$ to remove cell debris.

For preparation of the cell extracts, yeast colonies were seeded in YPDA medium, and harvested at $\mathrm{OD}_{600}$ 0.8-1.0. Cells were washed with sterile ice-cold water, lysed with a lysis buffer enriched by 1 volume of sterile glass beads, and briefly vortexed every $5 \mathrm{~min}$, for $1 \mathrm{~h}$. Lysates were cleared by centrifugation at $17000 \mathrm{~g}$ for $30 \mathrm{~min}$.

Protein concentration was measured by the Bradford assay. Solubilized proteins were resolved by $10 \%$ SDS-PAGE and transferred onto a nitrocellulose membrane. Blots were blocked in $0.1 \%$ Tween-20 and 5\% low-fat milk in phosphate-buffered saline (PBS) for $1 \mathrm{~h}$ and probed with anti-p53 antibody DO-1 at $4{ }^{\circ} \mathrm{C}$. Blots were developed with Dako peroxidase-conjugated rabbit anti-mouse immunoglobulin using the ECL chemiluminiscence detection kit (Amersham Biosciences, Vienna, Austria).

Fluorescence in situ hybridization (FISH). FISH was performed on tissue sections prepared from formalin-fixed, paraffin-embedded tissue blocks. Hybridization was performed with p53 locus-specific probe LSIp53 and centromeric probe CEP 17 (Vysis Inc., Downers Grove, IL, USA) according to the manufacturers' instructions. Images were scanned by a Leica DMRXA2 microscope equipped with CCD camera (COHU). Fluorescence signals were analyzed using Leica Q-FISH software (Leica Microsystems GmbH, Wetzlar, Germany).

\section{Results}

Assessment of the p53 protein level. To assess the p53 protein level in tumor tissue, we employed two approaches. Initially, 
we performed an immunohistochemical analysis of the tissue sections of formalin-fixed, paraffin-embedded tissue blocks using the p53-specific monoclonal antibody DO-7. We detected a strong p53 expression in 95-100\% of tumor cell nuclei (Fig. 1, panel a). Subsequently, we confirmed the high level of p53 in tumor tissue by immunoblotting using the p53-specific monoclonal antibody DO- 1 . At the same time, we found that the molecular weight of the tumor p53 protein was significantly higher than that of the standard p53 protein. The p53 protein of the standard molecular weight was present in the tumor tissue as well (Fig. 1, panel b).

FASAY. FASAY deduces the functional status of p53 from the color of colonies of transformed yeast cells. Yeast cells expressing functional p53 form large (round smooth) white colonies on the selection medium, while yeast cells expressing inactive p53 form small (round smooth) red colonies. The p53 status of an analyzed sample is assessed by calculating the ratio of red/white to the total colonies growing on the selection medium. Red colonies are equivalent to the tumor cells with mutant p53, and white colonies are equivalent to tumor and non-tumor cells with functional p53. The background frequency of the red colonies resulting predominantly from degraded mRNA and incorrectly assigned nucleotides during PCR typically does not exceed $10 \%$. In addition, FASAY can recognize the p53 mutations that only partially affect the transactivational potency of $\mathrm{p} 53$, for example, the temperaturedependent ones. In these cases, the phenotype of yeast colonies differs from the standard white and red colonies. The colonies are usually pink and reach a medium size due to the partial ability of the p53 protein to transactivate the reporter gene $(9,12)$.

To analyze the tumor tissue of our patient by FASAY, we first performed RT-PCR. When quality and quantity of the PCR product were tested by agarose gel electrophoresis, we noted that the molecular weight of the p53 PCR product prepared from tumor cells was markedly higher than the standard one (Fig. 2, panel a). Transformation of the yeast cells with this PCR product resulted in the formation of white $(6.8 \%)$, typical red $(4.0 \%)$ and unusual red colonies containing multiple white spots (89.2\%) (Fig. 2, panel b). These results suggest that the $\mathrm{p} 53$ gene of the patient underwent a mutation that did not completely impair its function.

Sequencing of the p53 cDNA. One of the significant advantages of FASAY is the detection of clonal p53 mutations even in cases when the fraction of cancer cells in analyzed tissue is too low for direct DNA sequencing. The tumor p53 cDNA can be recovered from transformed yeast cells and used for DNA sequencing (12-14). In this study, we inoculated four 'spotted' colonies, grew them separately in cultivation media overnight, and used them for DNA preparation. Purified DNA was then used for amplification of the p53 cDNA by PCR. Agarose gel electrophoresis of the amplified cDNA revealed that mobility of the PCR products predominantly corresponded to the p53 of standard molecular weight (Fig. 3, panel a). A low amount of the PCR products of larger molecular weight corresponding to the size of the PCRamplified p53 cDNA that was purified directly from tumor tissue was also detected (Fig. 3, panel a). Therefore, we did

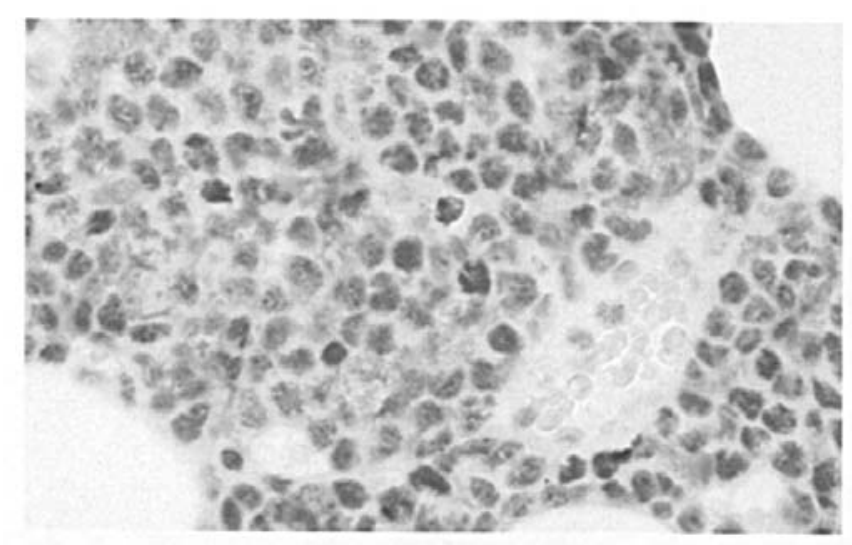

(a)

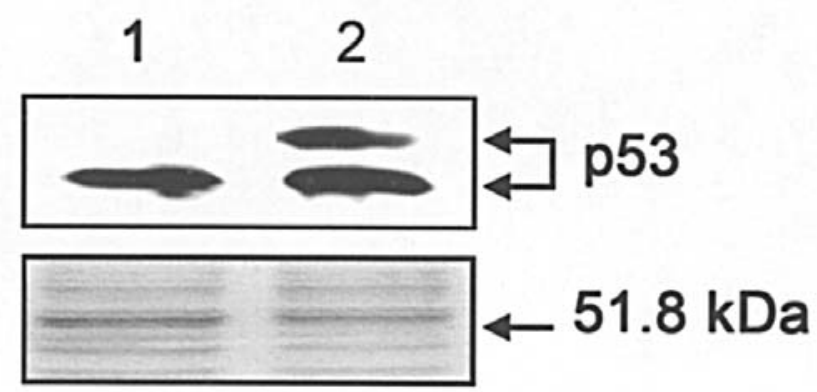

(b)

Figure 1. Assessment of the p53 protein level in tumor tissue. (a) Immunohistochemical detection of the p53 protein using monoclonal antibody DO-7 in a section of tumor tissue prepared from paraffin-embedded tissue block. (b) Protein extracts of the human breast carcinoma-derived cell line BT474 (1, positive control) and tumor tissue (2) were resolved by SDSPAGE and either immunoblotted using the p53-specific monoclonal antibody DO-1 (upper box) or stained with Coomassie brilliant blue (lower box).

not analyze the p53 mutation by DNA sequencing of the cDNAs prepared from the individual yeast colonies. Instead, we sequenced the p53 cDNA isolated directly from the tumor tissue (Fig. 2, panel a), and found the $30 \mathrm{bp}$ duplication g.12155_12184dup30 (genomic sequence X54156, GenBank) (Fig. 4) corresponding to c.366_395dup30 and leading to the repeat of 10 amino acids (Pro-Ala-Ala-Pro-Thr-Pro-Ala-AlaPro-Ala) in the position 77 to 86 in the p53 protein.

Analysis of the p53 status in yeast colonies. Each individual yeast colony obtained by FASAY represents a clone that produces one variant of p53 originating from analyzed tissue or raising during the RT-PCR process. Therefore, the origin of different PCR products made by amplification of the p53 cDNA of the individual 'spotted' yeast colonies was unclear (Fig. 3, panel a). To understand this result in more detail, we performed further analyses of the p53 status in the yeast cells forming the 'spotted' colonies. We grew the cells in cultivation medium, harvested them, and protein lysates were subjected to SDS-PAGE and immunoblotting using the p53-specific monoclonal antibody DO-1. We detected exclusively the p53 protein of the standard size in each sample (Fig. 3, panel b). Interestingly, when the yeast cells cultivated in liquid media 


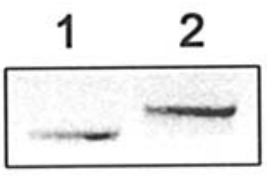

(a)

Day 3

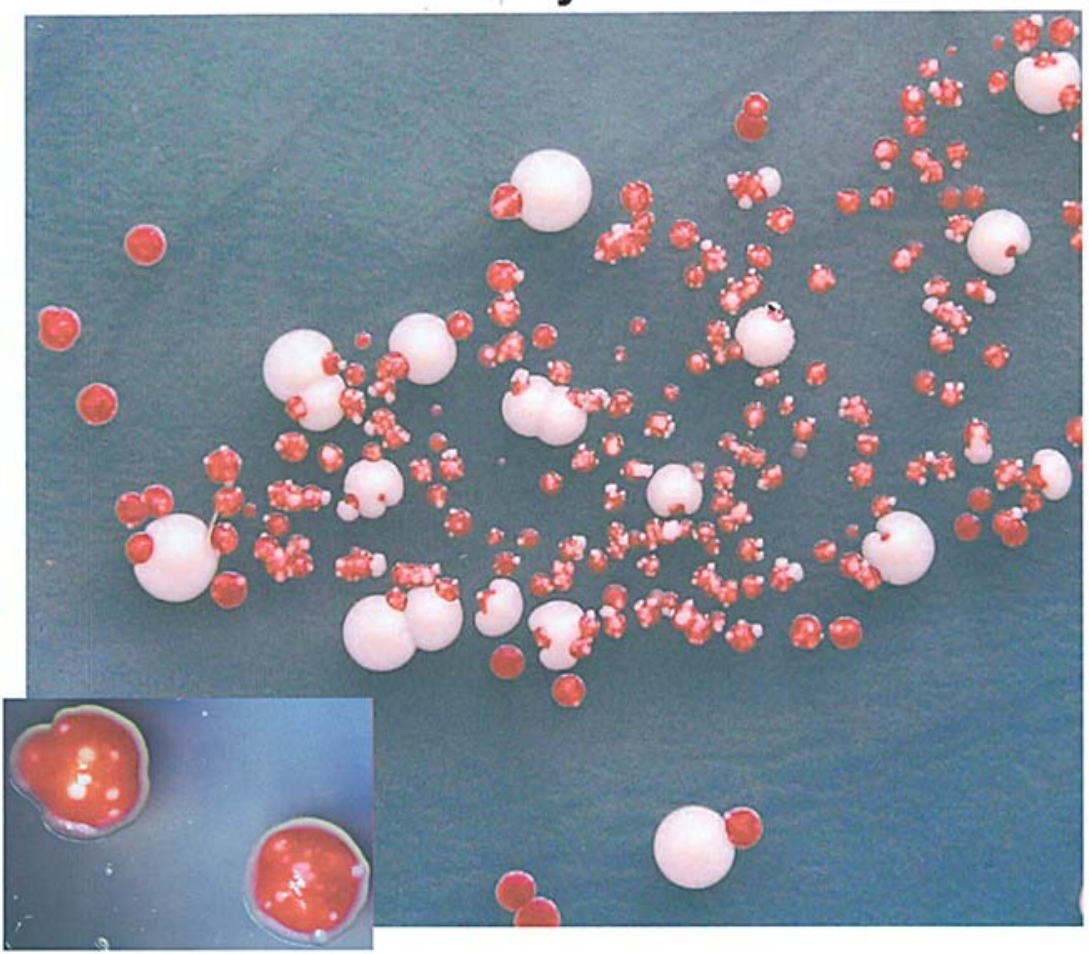

\section{Day 5}

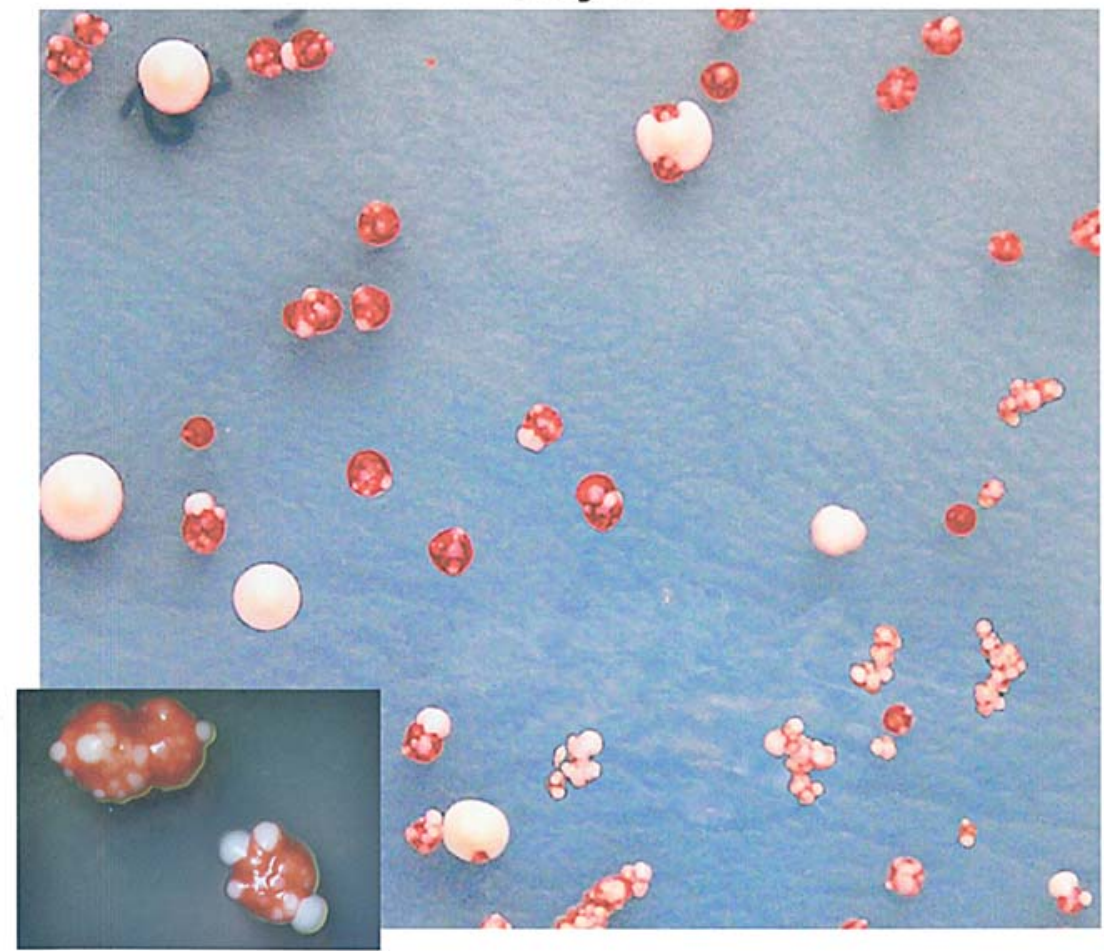

(b)

Figure 2. Analysis of the p53 function by FASAY. (a) p53 cDNA derived from the wild-type p53 control (1) and tumor tissue (2) were amplified by RT-PCR, resolved by agarose gel eletrophoresis and visualized by ethidiumbromide. (b) Competent yeast cells yIG397 were transformed with cDNA (2), plated on selection media and cultivated for the indicated periods. The morphology and color of the colonies are shown. Representative 'spotted' colonies are shown in the lower left corners at a higher magnification. 


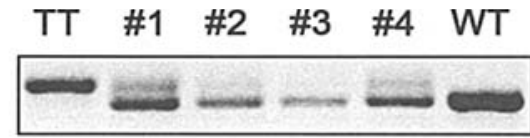

(a)

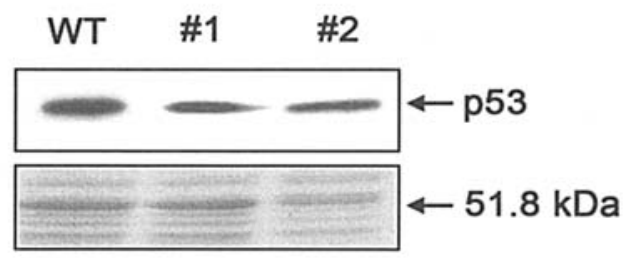

(b)

Figure 3. Analysis of the p53 status in 'spotted' yeast colonies. (a) The p53specific PCR products were amplified from DNA isolated from four individual yeast colonies (no.1-4), resolved by agarose gel electrophoresis and stained by ethidiumbromide. As controls, yeast cells expressing wildtype 533 cDNA (WT), and tumor tissue-derived RT-PCR product (TT) were used. (b) Protein extracts of two individual yeast colonies were resolved by SDS-PAGE and either immunoblotted using the p53-specific monoclonal antibody DO-1 (upper box) or stained with Coomassie brilliant blue (lower box). As a positive control, the yeast cells expressing wild-type p53 were used (WT).

were plated back on the selection agar medium, they solely formed the typical large white colonies adequate to cells expressing fully functional p53.

Fluorescence in situ hybridization. To determine the p53 status of the tumor tissue, we performed interphase FISH on tissue sections prepared from the formalin-fixed, paraffinembedded tissue blocks. We first performed FISH using the p53 locus-specific probe. We analyzed 65 cell nuclei and detected loss of one signal in 7 of them $(10.8 \%)$. Then, we performed FISH using the p53 locus-specific and $\mathrm{CH} 17$ centromeric probes. We analyzed 43 cell nuclei, all of which had two centromeric signals. Thirty-five nuclei $(81.4 \%)$ provided two p53 locus-specific signals, and 8 nuclei (18.6\%) provided only one p53 locus-specific signal.

\section{Discussion}

In contrast to other tumor suppressor genes, the majority of p53 mutations are missense substitutions. They represent $\sim 80 \%$ of all mutations detected in different types of cancer. The majority occur in the DNA-binding domain-coding region. Less frequent are other types of p53 mutations, such as insertions, short deletions and non-sense mutations that usually affect 5'- and 3'-termini of the p53 gene $(6,15-17)$. In this study, we describe a unique p53 mutation found in tumor tissue of the patient suffering from Burkitt's lymphoma, which is the $30 \mathrm{bp}$ duplication located outside the DNA-binding domain-coding region and which comprises of the fragment limited by codons 77 and 86 . To analyze the mutation in detail, we employed several different approaches. Immunohistochemical analyses showed a high p53 protein level in the tumor tissue, strongly suggesting the presence of p53 mutation. In addition, immunoblotting revealed that the tumor tissuederived p53 protein possesses an unusually high molecular weight. FASAY provided independent evidence that the p53 gene of the tumor tissue is enlarged. Electrophoretic mobility of the band representing the product of RT-PCR prepared from tumor tissue during FASAY was markedly retarded in comparison with mobility of the RT-PCR product of standard p53. Sequencing of the tumor tissue-derived p53 cDNA revealed that the p53 molecule is enlarged due to the $30 \mathrm{bp}$ duplication of the fragment comprising of codons 77 to 87 . FASAY unambiguously showed that the tumor tissue-derived p53 is not fully functional. Frequency of the positive 'spotted' colonies reached $90 \%$ suggesting that the second p53 allele is absent from tumor cells. However, FISH analysis failed to confirm loss of p53 heterozygosity. Most of the tumor cell nuclei were markedly positive for two p53-specific signals. Using the chromosome 17-centromeric and p53 locus-specific probes, we excluded the hypothesis that the tumor cells may be tetraploid. Another version of FISH that focused on $\mathrm{t}(8 ; 14)(\mathrm{q} 24 ; \mathrm{q} 32)$, did not show the tetraploid status of tumor cells. Thus, we can only speculate as to what occurred with the second allele of the p53 gene in tumor cells. There are three options. i) The non-mutated p53 allele may be physically present, thus providing the positive FISH signal, but silenced, thus providing no signal in FASAY (RT-PCR). The silencing can be accomplished either genetically, for example, by promoter mutation or epigenetically, for example, by promoter methylation. ii) The p53 allele may not be silenced, but rather mutated in a way that interferes with its detection by RT-PCR of FASAY. This may result from a point mutation located in the region of primer annealing. This option would explain the presence of the standard-sized p53 protein in tumor tissue (Fig. 1, panel b) and we consider it as the most likely. iii) The same mutation (the 30 bp duplication) may be present in the two p53 alleles. This option is rather unlikely, because the probability that the same mutation occurs twice independently,

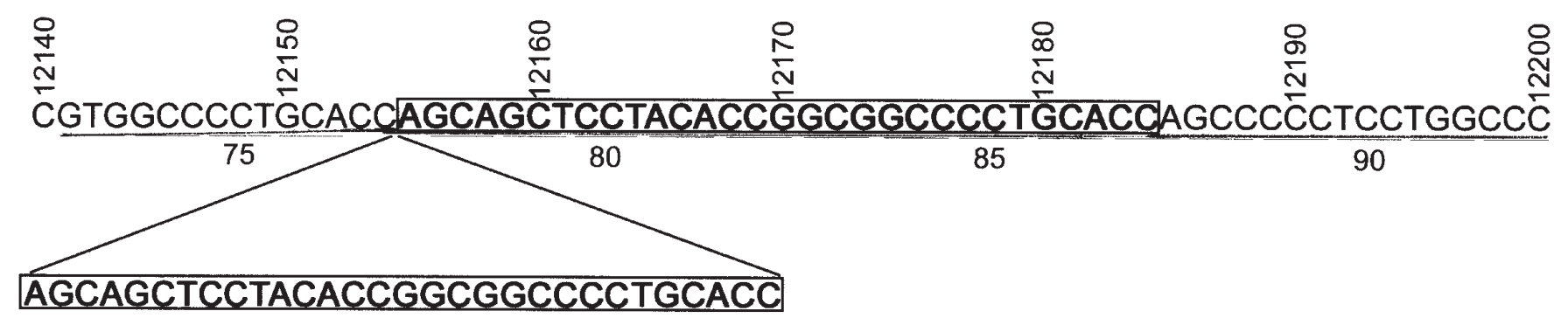

Figure 4. Duplication of the $30 \mathrm{bp}$ region in nucleotide sequence of the p53 gene derived from tumor tissue. The upper numbers mark the nucleotide positions of genomic DNA and the lower numbers indicate positions of the codons; marked by horizontal bars. The boxed sequences show duplicated regions. 
is extremely low. However, gene conversion or mitotic recombination may be considered.

The morphology of the 'spotted' yeast colonies produced in FASAY is very unusual. Similarly, the behavior of the yeast cells as revealed by analysis of the p53 status is unique. Thus far, we have routinely confirmed the same properties of the p53 genes/proteins in tumor and transformed yeast cells (18). In this study, the p53 cDNA derived from tumor cells was larger than the yeast-derived p53 cDNA. On the protein level, the enlarged variants of the tumor-derived p53 were completely lost by transformed yeast cells. We suppose that this results from instability of the $30 \mathrm{bp}$ insertion in yeasts. The yeast cells transformed with the duplication-containing p53 cDNA formed small red colonies in FASAY. This indicates the non-functional status of p53. However, during extended cultivation, the morphology of these colonies changes. Within three days of cultivation, the tiny white spots appeared on the surface of the colonies, and they grew rapidly during the next cultivation (Fig. 2, panel b). We hypothesize that these spots represent subclones of the yeast cells that lost the duplicated fragment of p53 cDNA thus resuming the p53 function. The cells with functional p53 acquired a growth advantage because they can form adenine on their own. Therefore, they gradually overgrow the cells with the p53 mutation that are limited by a low content of adenine in the selection media (9). This is manifested by enlarging white spots on the surface of red yeast colonies. For the purification of p53 cDNA from transformed yeast cells, a short cultivation time and small volume of media are used. These conditions are permissive for presence of the residual tumor p53 cDNA variants as detected by agarose gel electrophoresis (Fig. 3, panel a). For analyses of the p53 protein produced by yeasts, longer cultivation periods and larger volumes of media are required. Therefore, duplication may be lost under these conditions and only the p53 protein of common molecular weight can be detected by immunoblotting (Fig. 3, panel b).

Functional assays in yeast have been widely used for analysis of the p53 status, and they are generally considered as very reliable. The presented unusual case implicates possible limitations for using the yeast cells for analyses of the human p53 gene. We suggested that some p53 mutations are unstable in yeast cells. The method itself disclosed the presence of inactivating p53 mutations unambiguously and reproducibly, while the unusual morphology of the yeast colonies indicated an unusual property of $\mathrm{p} 53$. This provides further evidence that FASAY is the method of choice for functional analyses of $\mathrm{p} 53$.

\section{Acknowledgements}

We thank Richard Iggo for providing vector pSS16 and yeast strain yIG397. This work was supported by grant NR/9305-3 of the Internal Grant Agency of the Ministry of Health of the Czech Republic.

\section{References}

1. Drexler HG, Fombonne S, Matsuo Y, Hu ZB, Hamagichi H and Uphoff CC: p53 alterations in human leukemia-lymphoma cell lines: in vitro artifact or prerequisite for cell immortalization? Leukemia 14: 198-206, 2000

2. Lindstrom MS and Wiman KG: Role of genetic and epigenetic changes in Burkitt lymphoma. Semin Cancer Biol 12: 381-387, 2002 .

3. Wilda M, Bruch J, Nardet L, Rawer D, Reiter A, Borkhardt A and Woessmann W: Inactivation of the ARF-MDM-2-p53 pathway in sporadic Burkitt's lymphoma in children. Leukemia 18: 584-588, 2004

4. Levine AJ, Hu W and Feng Z: The p53 pathway: what questions remain to be explored? Cell Death Differ 13: 1027-1036, 2006.

5. Olivier M, Eeles R, Hollstein M, Khan MA, Hartus CC and Hainaut P: The IARC TP53 database: new online mutation analysis and recommendations to users. Hum Mutat 19: 607-614, 2002

6. Soussi T, Asselain B, Hamroun D, Kato S, Ishioka C, Claustres M and Beroud C: Meta-analysis of the p53 mutation database for mutant p53 biological activity reveals a methodological bias in mutation detection. Human Cancer Res 12: 62-70, 2006.

7. Yang Y, Li CCH and Weismenn AM: Regulating the p53 system through ubiquitination. Oncogene 23: 2096-2106, 2004.

8. Ishioka C, Frebourg T, Yan YX, Vidal M, Friend SH, Schmidt S and Iggo R: Screening patients for heterozygous p53 mutations using a functional assay in yeast. Nat Genet 5: 124-129, 1993.

9. Flaman JM, Frebourg T, Moreau V, Charbonnier F, Martin C, Chappuis P, Sappino AP, Limacher JM, Bron L, Benhattar J, Tada M, Van Meir EG, Estreicher A and Iggo RD: A simple p53 functional assay for screening cell lines, blood, and tumors. Proc Natl Acad Sci USA 92: 3963-3967, 1995.

10. Trkova M, Torerova L, Kodet R, Hedvicakova P and Sedlacek Z: A Li-Fraumeni syndrome family with retained heterozygosity for a germline TP53 mutation in two tumors. Cancer Genet Cytogenet 145: 60-64, 2003.

11. Smardova J, Nemajerova A, Trbusek M, Vagunda V and Kovarik J: Rare somatic p53 mutation identified in breast cancer: a case report. Tumor Biol 22: 59-66, 2001.

12. Pavlova S, Mayer J, Koukalova H and Smardova J: High frequency of temperature-sensitive mutations of p53 tumor suppressor in acute myeloid leukemia revealed by functional assay in yeast. Int J Oncol 23: 121-131, 2003.

13. Nenutil R, Smardova J, Pavlova S, Hanzelkova Z, Miller P, Fabian P, Hrstka R, Janotova P, Radina M, Lane DP, Diates PJ and Vojtesek B: Discriminating functional and non-functional p53 in human tumours by p53 and MDM2 immunohistochemistry. J Pathol 207: 251-259, 2005.

14. Trbusek M, Malcikova J, Smardova J, Kuhrova V, Mentzlikova D, Francova H, Bukovska S, Svitakova M, Kuglik P, Linkova V, Doubek M, Brychtova Y, Zacal J, Kujickova J, Pospísilova S, Dvorákova D, Vorlícek J and Mayer J: Inactivation of p53 and deletion of ATM in B-CLL patients in relation to IgVH mutation status and previous treatment. Leukemia 20: 1159-1161, 2006.

15. Hainaut P and Hollstein M: p53 and human cancer: the first ten thousand mutations. Adv Cancer Res 77: 81-137, 2000.

16. Olivier M, Hussain SP, de Fromentel CC, Hainaut P and Harris CC: TP53 mutation spectra and load: a tool for generating hypotheses on the etiology of cancer. IARC Sci Publ 157: 247-270, 2004.

17. Petitjean A, Achatz MIW, Borresen-Dale AL, Hainaut P and Olivier M: TP53 mutations in human cancers: functional selection and impact on cancer prognosis and outcomes. Oncogene 26: 2157-2165, 2007.

18. Smardova J, Pavlova S, Svitakova M, Grochova D and Ravcukova B: Analysis of p53 status in human cell lines using a functional assay in yeast: Detection of new non-sense p53 mutation in codon 124. Oncol Rep 14: 901-907, 2005. 